Chinese Journal of Electronics

Vol.XX, No.X, Jan. 2017

\title{
Saliency-based Segmentation of Optic Disc in Retinal Images
}

\author{
ZOU Beiji ${ }^{1}$, LIU Qing ${ }^{1}$, YUE Kejuan ${ }^{1,2}$, CHEN Zailiang ${ }^{1}$, CHEN Jie ${ }^{3}$, ZHAO Guoying ${ }^{3}$ \\ (1. School of Information Science and Engineering, Central South University, Changsha, 410083, China) \\ (2. Hunan First Normal University, School of Information Science and Engineering, Changsha 410205, China) \\ (3. Center for Machine Vision and Signal Analysis, University of Oulu, Oulu, 90570, Finland)
}

\begin{abstract}
Accurate segmentation of optic disc (OD) is significant for the automation of retinal analysis and retinal diseases screening. This paper proposes a novel optic disc segmentation method based on the saliency. It includes two stages:optic disc location and saliency-based segmentation. In the location stage, the OD is detected using a matched template and the density of the vessels. In the segmentation stage, we treat the OD as the salient object and formulate it as a saliency detection problem. To measure the saliency of a region, the boundary prior and the connectivity prior are exploited. Then geodesic distance to the window boundary is computed to measure the cost the region spends to reach the window boundary. After a threshold and ellipse fitting, we obtain the OD. Experimental results on two public databases for OD segmentation show that the proposed method achieves thestate-of-the-art performance.
\end{abstract}

Key words - Retinal fundus, Optic disc segmentation, Boundary prior, Region connection, Saliency measure.

\section{Introduction}

Nowadays, glaucoma, diabetic retinopathy, eye hypertension and macular degeneration become the most common causes of visual impairment and blindness ${ }^{[1]}$. To discover those retina related diseases early, potential patients are highly suggested to have regular inspections of the retinal fundus, the unique part of the body where the microcirculation can be observed directly. However, the large population base and rapid speed of ageing contribute to the increasing number of retinal diseases which in turn greatly increases the workload of the ophthalmologists. To reduce the labour intensity of the ophthalmologists, the automation of retinal image analysis and retinal diseases screening become emergent.

For the screening system, one of the most significant procedure is the optic disk (OD) segmentation. The OD in the retinal image always appears as bright yellowish and elliptical region, from which low intensity vessels extend towards and around the fovea in a radial fashion curving. On one hand, the OD provides important manifestations of the presence of glaucoma and hypertension. For example, the cup-to-disk ratio is used for the assessment of the progress of glaucoma ${ }^{[2,3]}$. On the other hand, the location of the OD facilitates the detection of fovea ${ }^{[4]}$, exudate segmentation ${ }^{[5]}$, etc.

Numerous works about the OD segmentation have been proposed in the literature. In general, existing OD segmentation methods take the shape prior and the appearance prior of the OD into consideration. For example, Lalonde et al. first obtain the candidate regions using a pyramid of Haar-based discrete wavelet transform, then compute the Hausdorff distance between the edge map of the candidate region and a circular template to search the OD contour ${ }^{[6]}$. In [7], Lowell et al. propose to use an invariant of $\mathrm{Hu}^{\prime}$ circular deformable mode ${ }^{[9]}$ to segment the OD after obtaining the location of the OD by filtering with a specialised template. Roychowdhury et al. directly use an ellipse to fit the convex hull of the candidate region which is obtained by a learnt classification ${ }^{[10]}$. In [11], region-based active contour model is proposed to improve the segmentation on the range of OD instances, in which rich features including the red colour channel and texture representations by Gaussian filter and Gabor filter are used. The snake combining smoothness, gradient magnitude and orientation, intensity information are proposed, in which the edge points at each iteration are updated according to their neighbours ${ }^{[12]}$. In [13], the ves-

* Manuscript Received XX XX, 2017; Accepted XX XX, 2017. This work was supported by the scholarship from China Scholarship Council (CSC), the National Natural Science Foundation of China (No.61573380, No. 61440055 and No. 61672542), and the Academy of Finland, Tekes Fidipro Program and Infotech Oulu.

(C) 2017 Chinese Institute of Electronics. DOI:10.1049/cje.20XX.0X.0XX 


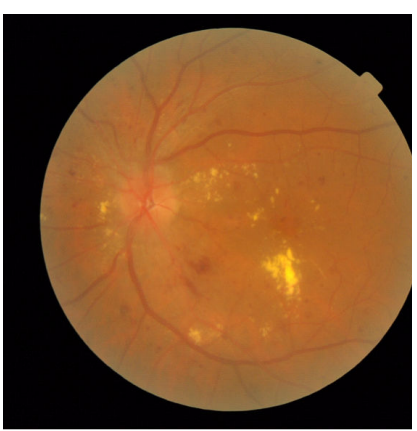

(a)

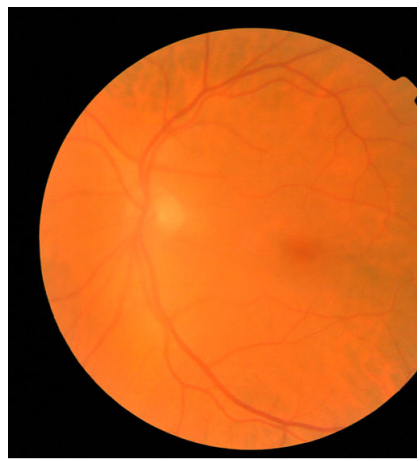

(e)

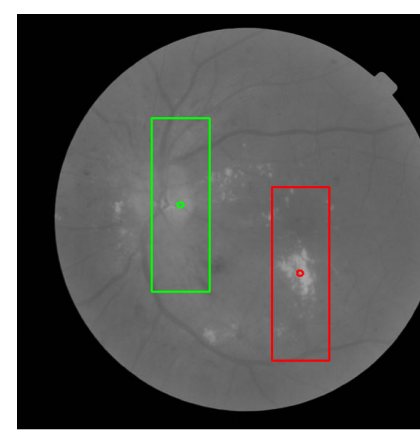

(b)

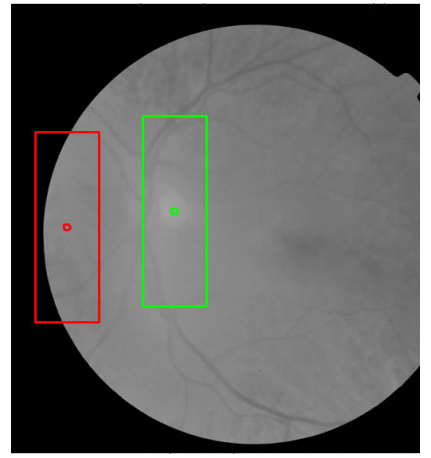

(f)

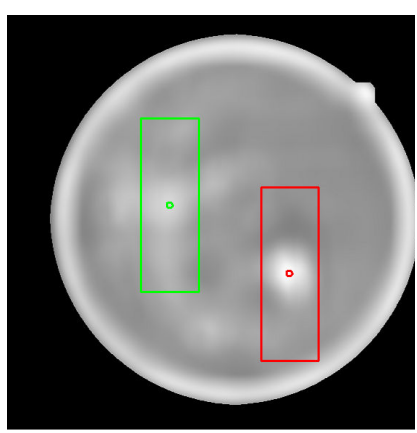

(c)

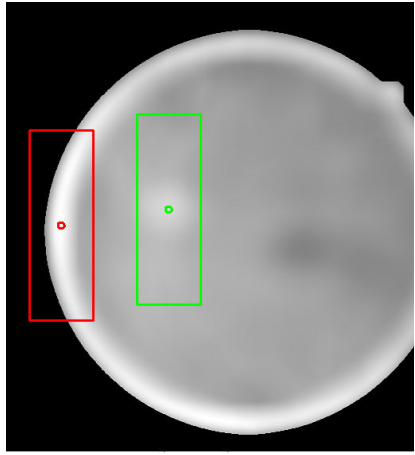

(g)

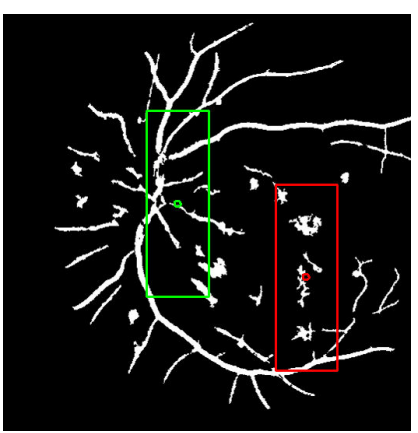

(d)

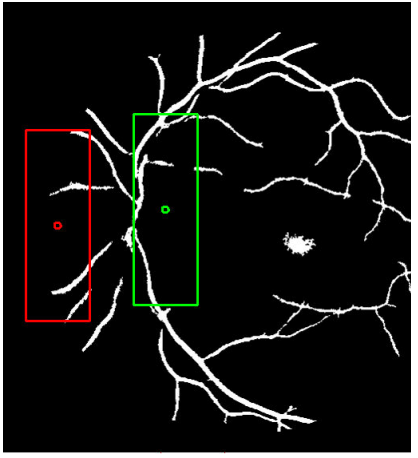

(h)

Figure 1: Examples for the OD location. (a) \& (e): the input retinal images; (b) \& (f): the intensity channel of the retinal image; (c) \& (g): the response images after convolution with the matched filter proposed by Lowell et al. [7]; (d) \& (h): the vessel extracted from the retinal images. The OD locations obtained by Lowell et al. [7] are marked in red circles while the OD locations obtained by the proposed method are marked in green circles.

sels in the retinal image are first segmented using graph cut to localise the OD. Then two alternative methods are proposed to segment the OD. One is the Markov random filed (MRF) image reconstruction which removes the vessels from the optic disk. The other is the compensation factor method which incorporates the vessel information into the graph cut formulation.

Those methods mainly focus on what the OD looks like but ignore the information the background provides. In this paper, we take both the priors about the OD and the background into consideration. First, we localise the $\mathrm{OD}$ in the retinal image by answering the question about what the OD looks like. In this stage, the response of a matched template is used to obtain the candidate location of the OD, and the vessel density is computed for the validation. Then we treat the OD as the salient object in the OD window and formulate problem of the OD segmentation as a problem of the salient object detection. A saliency map is generated to indicate the probability of a pixel belonging to the OD by answering the question about what the background looks like. To this end, two priors about the background regions are exploited. One is the boundary prior which assumes that the regions on the boundary of the OD window are background. This is definitely true since the OD is located near the centre of the OD window. The other is connectivity prior which assumes that the background regions are smooth. So it is much easier for the background regions to reach the window boundary than the OD regions. The difficulty degree of a region reaching to the window is defined as saliency by Wei et al. ${ }^{[14]}$. The less salient a region is, the more likely it belongs to the background. In turn, the more salient a regions is, the more likely it belongs to the OD. Finally, by simple thresholding and ellipse fitting, we obtain the OD. Note that though these two background priors are widely used in salient object detection ${ }^{[15,16]}$, they are still novel to the OD segmentation.

In the remainder of this paper, we detail the proposed method in Section II. Then we report the experimental results in Section III. The final section concludes this paper.

\section{The Proposed Method}

The proposed method includes two main processing phases: 1) the OD location phase to localise the centre of the OD; 2) the OD segmentation phase to segment the OD based on the saliency estimation.

\section{OD location}

The OD only accounts for a small part of the whole 


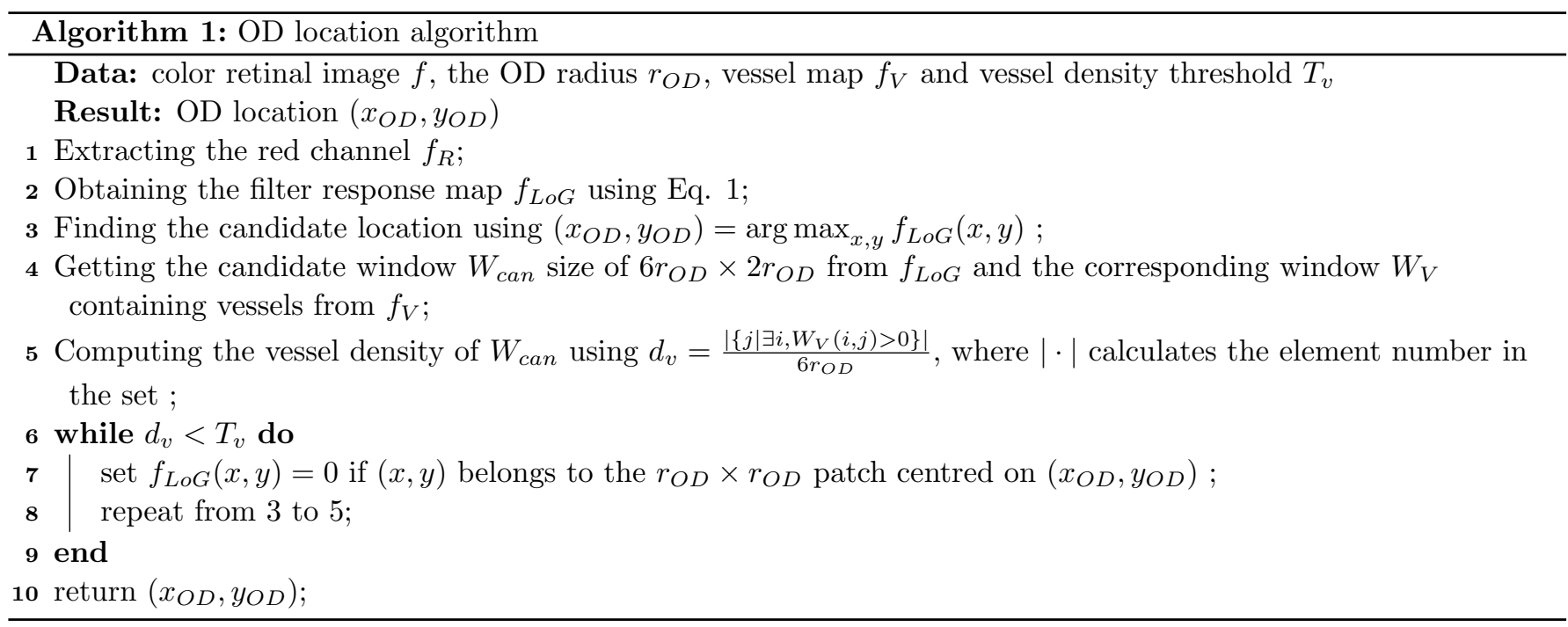

retinal image. Thus estimating the location of the OD before the segmentation eliminates the adverse affects from hard exudates, cotton wool spot and bright fringe etc. In the retinal image, the OD is characterised as a high intensity near-circular disk and low intensity blood vessels extending towards and around the fovea in a radial fashion curving. Motivated by the characteristics of the optic disk, the $R G B$ retinal image is first converted into $H S I$ image and the intensity component $f_{I}$ is extracted. Then we filter $f_{I}$ with the matched filter proposed by Lowell et al. ${ }^{[7]}$ :

$$
f_{L o G}(x, y)=f_{I}(x, y) \otimes(-\operatorname{LoG}(x, y))
$$

where $\otimes$ is the convolution operator and $\operatorname{LoG}(x, y)$ is the Laplacian of Gaussian filter:

$$
\operatorname{LoG}(x, y)=\frac{x^{2}+y^{2}-2 \sigma^{2}}{\sigma^{4}} \exp \left(-\frac{x^{2}+y^{2}}{2 \sigma^{2}}\right)
$$

In the response map $f_{L o G}$, the OD is enhanced. However, some regions, such as the large exudate (see Fig. 1(c)), the high contrast fringes (see Fig. 1(g)) also are enhanced. To distinguish the OD from those regions, the vessel density is computed. First, the location $\left(x_{O D}, y_{O D}\right)$ with maximum response is selected as the candidate OD location. Then, to validate whether the candidate location is on the OD or not, the main vessels are extracted by thresholding the difference of diffusion map ${ }^{[8]}$ with Otsu threshold method. A $6 r_{O D} \times 2 r_{O D}$ window $W_{\text {can }}$ centred on $\left(x_{O D}, y_{O D}\right)$ is extracted, and the vessel density $d_{v}$ is computed. If its vessel intensity is greater than $T_{v}$ which is set to $0.8 \times 6 r_{O D}$ in the experiments, it is selected as the OD location. Otherwise, we set the response value at $\left(x_{O D}, y_{O D}\right)$ as well as the pixels around it as zeros, and validating the next candidate OD location until the vessel density of the candidate location is greater than $T_{v}$. This procedure is detailed in Algorithm. 1. Fig. 1 shows the effectiveness of the proposed OD location method.

\section{Saliency - based OD segmentation}

(1) Preprocessing

To eliminate the adverse affect from other bright structures such as the exudates and cotton wool spots, only a $5 r_{O D} \times 5 r_{O D}$ window $W$ containing the OD and centred on $\left(x_{O D}, y_{O D}\right)$ is considered. As previous methods suggested, preprocessing is necessary prior to OD segmentation for a more robust segmentation. The blood vessels bring difficulties in accurate OD segmentation. Thus before the OD segmentation, the vessels in the OD window $W$ are removed using group-based sparse representation ${ }^{[16]}$ followed by two close-open filtering. Similar to [17], for an OD window whose red channel is not saturated, we perform preprocessing in the red channel since the OD always appear with the high contrast against its surroundings. For an OD window whose red channel is saturated, it becomes difficult to segment the OD from its surrounding background. In this case, we replace the red channel with the intensity component, then perform preprocessing.

\section{(2) Saliency-based OD segmentation}

After the vessels are removed, there are two ways to segment the OD. One is to directly segment it from the OD window based on the appearance and shape priors about the OD, which is widely adopted in the previous methods. The other one is to estimate the background according to the background priors, then in turn to segment the OD. In this paper, the second way is adopted because the priors about background here are more robust.

As we have mentioned before, two priors can be observed in the OD window $W$ :

- the boundary prior: the regions on the OD window boundary are background because the OD is located 


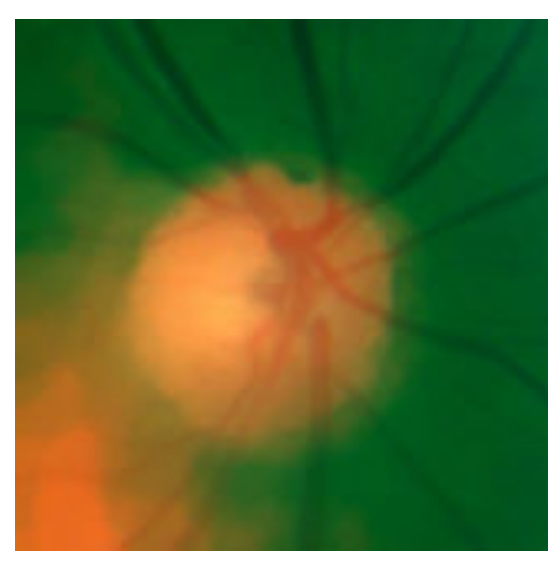

(a)

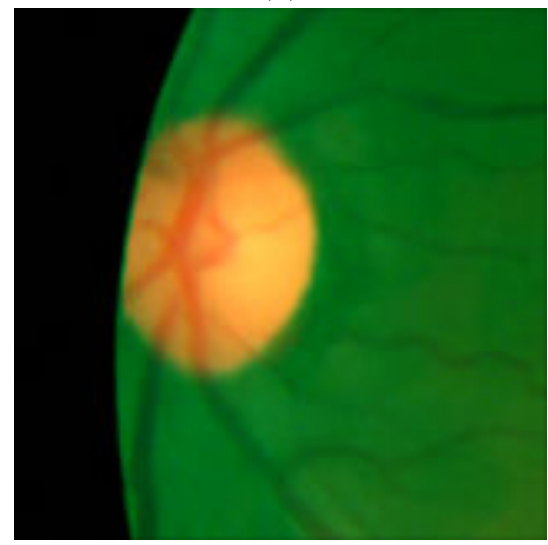

(d)

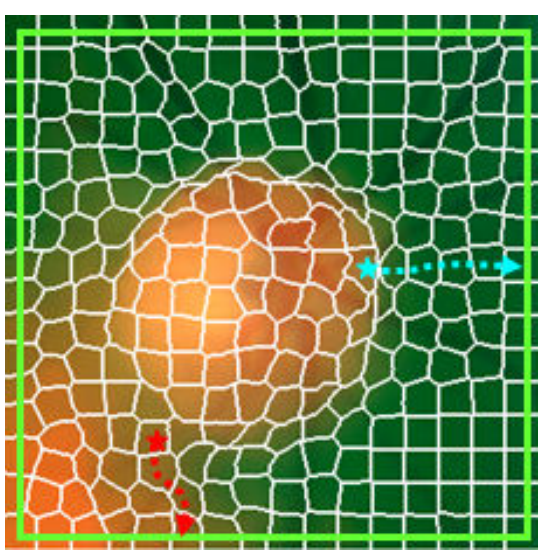

(b)

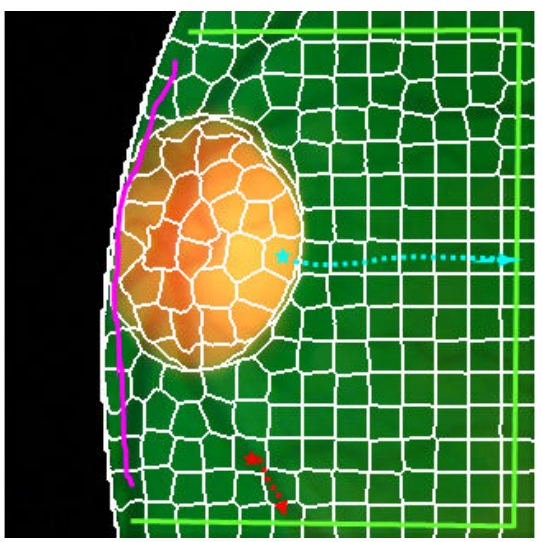

(e)

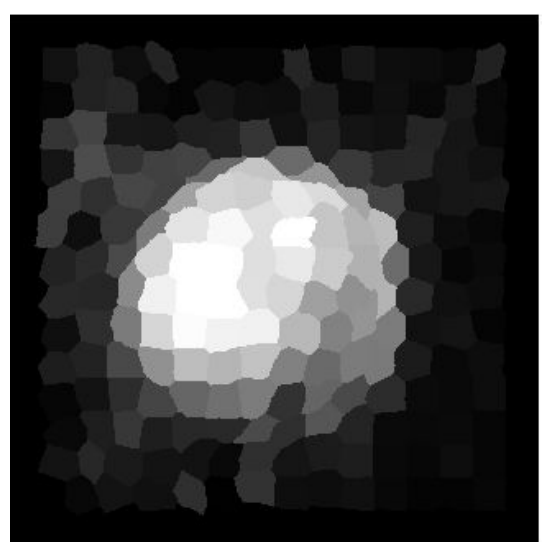

(c)

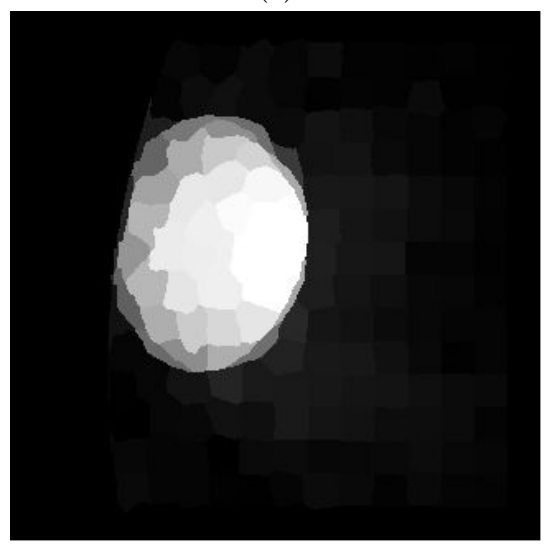

(f)

Figure 2: Examples for boundary prior and connectivity prior. (a) and (d) are the OD window. (b) and (e) are the results of oversegmentation. For the purpose of clarity, the size of superpixels is about 200 pixels while in our experiment it is set to 5 . It is obvious that all of the superpixels in $P_{B}$, i.e., the green lines go through, are background while the superpixel set $P_{F}$, i.e., the violet line goes through, contains both background and OD superpixels. Examples for the shortest paths of the superpixels to the window boundary are showed in dotted lines. For the background superpixels such as those the red stars locate, they can reach the window boundary easily due to the smoothness of the background regions. But for the OD superpixels such as those the cyan stars locate, the costs to reach the window boundary are high due to the abrupt changes between the OD and the background. (c) and (f) are the saliency maps.

near the centre of the OD window $W$;

- connectivity prior: the background regions in the OD window can easily reach to the window boundary because of their high smoothness while the OD regions are difficult to reach the window boundary since they are located near the window centre and always brighter than the background.

Leveraging these two priors about the background, the geodesic distance to the image boundary is proposed to estimate the saliency of the region, which reflects the probability of a superpixel belonging to OD and in turn facilitates the OD segmentation.

For the OD window, only the region in FOV is considered. First, it is decomposed into superpixels using
SLIC ${ }^{[18]}$, denoted by $p_{1}, \cdots, p_{M}$. These superpixels are divided into three disjoint subsets: (a) the superpixels located on the OD window boundary, denoted as $P_{B}$ (e.g. the superpixels the green lines go through in Fig. 2(b) and Fig. 2(e)); (b) the superpixels which are adjacent to the FOV mask boundary, denoted by $P_{F}$ (e.g. the superpixels the violet lines go through in Fig. 2(e)); (c) the rest superpixels which are located in the FOV, denoted by $P_{i n}$. Then we build an undirected weighted graph $\mathcal{G}=\{\mathcal{V}, \mathcal{E}\}$, where $\mathcal{V}=\left\{p_{1}, \cdots, p_{M}\right\}$ is the superpixel set and $\mathcal{E}$ is the edge set. There are two kinds of edges:

\section{(a) Internal edges}

Any two adjacent superpixels in $P_{i n} \cup P_{F}$ is connected by an edge. Each superpixel is represented by its average $L a b$ colour since the $L a b$ colour space is designed to 
approximate human vision. The edge weight is defined as the appearance dissimilarity between two adjacent superpixels which is measured by the Euclidean distance between their average colour features. The more different two adjacent superpixels, the larger the weight of the edge connecting them is.

(b) Edges between the superpixels in $P_{F}$ and $P_{B}$

Since the OD can be located on the boundary of FOV (see Fig. 2(d)), the superpixels located on the boundary of the FOV can not be regarded as background directly. To increase the connectivity between these superpixels and the superpixels on the OD window, edges are added between the superpixels in $P_{F}$ and $P_{B}$. Similarly, their edge weights are computed in the same way as computed for the internal edges.

To estimate the saliency for each superpixel $p_{i}$ in $\left\{P_{F} \cup P_{i n}\right\}$, the geodesic distance from $p_{i}$ to the boundary superpixel set $P_{B}$ is computed:

$$
\operatorname{Sal}\left(p_{i}\right)=\min d\left(p_{i}, p_{j}\right) \text { s.t. } p_{j} \in P_{B}
$$

where $d\left(p_{i}, p_{j}\right)$ is the accumulated weights along the shortest path from superpixel $p_{i}$ to superpixel $p_{j} \in P_{B}$ :

$$
\begin{aligned}
& d\left(p_{i}, p_{j}\right)=\min _{p_{1}=p_{i}, p_{2}, \cdots, p_{n}=p_{j}} \sum_{k=1}^{n-1} \operatorname{cost}\left(p_{k}, p_{k+1}\right) \\
& \text { s.t. }\left(p_{k}, p_{k+1}\right) \in \mathcal{E}
\end{aligned}
$$

where $n$ is the the length of the path from $p_{i}$ to $p_{j}$, and $\operatorname{cost}\left(p_{k}, p_{k+1}\right)$ is the edge weight.

After we obtain the saliency map Sal, the OD are highlighted from the window. A simple Otsu threshold operator is used to obtain the binary OD segmentation. As the OD region exhibits as an ellipse shape, we fit the OD segmentation to an ellipse function to smooth the boundary of the segmented OD.

\section{Experiments and Results}

\section{Data sources}

The validation of the proposed method is carried out on two public databases: DRISONS ${ }^{[19]}$ and MESSIDOR $^{[20]}$. DRISONS${ }^{[19]}$ contains 110 retinal fundus images size of $600 \times 400$. Those images were collected from 55 patients, among which $23.1 \%$ patients had glaucoma and $76.9 \%$ patients had eye hypertension. Their boundaries are labelled by two specialists. $\operatorname{MESSIDOR}^{[20]}$ includes 1200 retinal fundus images, which were captured with a 45 degree field of view using eight bits per color planes at $1440 \times 960,2240 \times 1488$ or $2304 \times 1536$. Both normal images and diabetic retinopathy images are included. Each image was manually delimited by experts from the University of Huelva ${ }^{[20]}$.

\section{Evaluation metrics}

Following Morales et al. ${ }^{[20]}$, we use the following metrics to evaluate the proposed method: (1) Jaccard's coefficient JC: measuring the overlapping degree between the ground truth $A_{G}$ and the segmented OD region $A_{S}$ by $J C=\left|A_{G} \cap A_{S}\right| /\left|A_{G} \cup A_{S}\right| ;$ (2) Dice's coefficient DC: describing the similarity between $A_{G}$ and $A_{S}$ by $D C=2 \times\left|A_{G} \cap A_{S}\right| /\left(\left|A_{G}\right|+\left|A_{S}\right|\right) ;$ (3) Accuracy Ac: counting the fraction of pixels that are correctly classified; (4) True positive fraction TPF: computing the fraction of OD pixels that are corrected segmented; (5) False positive fraction FPF: computing the fraction of segmented pixels that are incorrectly segmented.

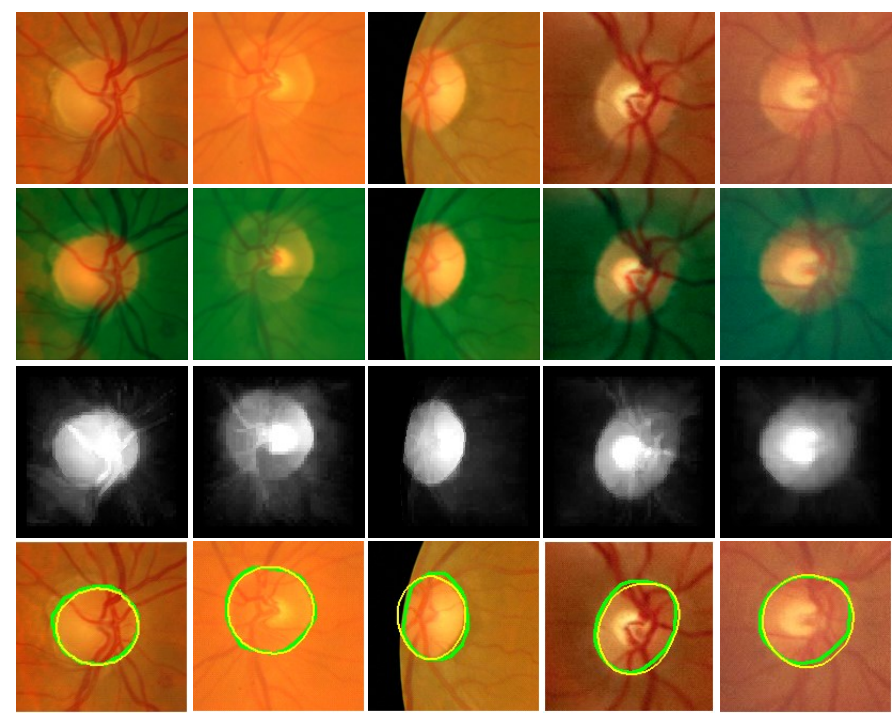

Figure 3: Examples for the OD segmentation. The 1st row: the input OD windows; the 2nd row: the results after preprocessing; the 3rd row: the saliency maps; the 4th row: the visualisation of the final results where the green contours are obtained by our method and the yellow contours are labelled by ophthalmologists.

\section{Results and comparisons}

To demonstrate the effectiveness of the proposed method, we test our method on two public datasets. Table. 1 and Table. 2 report the results and the comparisons to the previous methods on two datasets. On the DRISONS dataset, we take the OD mask labelled by the first specialist as ground truth, and the segmentations by the second specialist are also compared with the ground truth to obtain inter-expert differences, which is reported in the first row in Table. 1. From the two tables, it can be seen that the proposed method outperforms the previous methods. Especially, it achieves $88.15 \%$ of JC and $95.27 \%$ of TPF on DRISONS, which increases the JC by $3.90 \%$ and TPF by $2.46 \%$ comparing to Morales et al. [21]. On MESSIDOR, the proposed method achieves $84.83 \%$ of JC and $93.77 \%$ of TPF, which is better than Morales et al. [21] by $2.55 \%$ and $0.77 \%$ in terms of JC and TPF respectively. Morales et al. [21] oversegment the OD window using a stochastic watershed transformation, then discriminate the OD segment according to the mean intensity. 
Table 1. Comparisons with the state-of-the-art methods on the DRISONS dataset

\begin{tabular}{|c|c|c|c|c|c|}
\hline Methods & $\mathrm{JC}$ & $\mathrm{S}$ & Ac & TPF & FPF \\
\hline second observer & $0.9202(0.0455)$ & $0.9578(0.0265)$ & $0.9970(0.0017)$ & $0.9498(0.0537)$ & $0.0012(0.0009)$ \\
\hline Walter et al. [22] & $0.6227(0.3695)$ & $0.6813(0.3854)$ & $0.9689(0.0492)$ & $0.6715(0.3980)$ & $0.0210(0.0417)$ \\
\hline Morales et al. [21] & $0.8424(0.0074)$ & $0.9084(0.0982)$ & $0.9934(0.0051)$ & $0.9281(0.1177)$ & $0.0040(0.0041)$ \\
\hline Ours & $0.8815(0.0808)$ & $0.9348(0.0524)$ & $0.9960(0.0032)$ & $0.9527(0.0470)$ & $0.0026(0.0032)$ \\
\hline
\end{tabular}

Table 2. Comparisons with the state-of-the-art methods on the MESSIDOR dataset

\begin{tabular}{|c|c|c|c|c|c|}
\hline Methods & JC & S & Ac & TPF & FPF \\
\hline Morales et al. [21] & $0.8228(0.1384)$ & $0.8950(0.1056)$ & $0.9949(0.0050)$ & $0.9300(0.1239)$ & $0.0035(0.0041)$ \\
Yu et al. [17] & 0.83 & N.A & N.A & N.A & N.A \\
Roychowdhury et al.[10] & $0.8373(0.095)$ & N.A & $0.9956(0.001)$ & $0.9043(0.072)$ & $0.0018(0.0008)$ \\
Ours & $\mathbf{0 . 8 4 8 3 ( \mathbf { 0 . 1 2 0 4 } )}$ & $\mathbf{0 . 9 1 1 9 ( \mathbf { 0 . 0 9 4 6 ) }}$ & $\mathbf{0 . 9 9 8 1 ( \mathbf { 0 . 0 0 2 1 } )}$ & $\mathbf{0 . 9 3 7 7}(\mathbf{0 . 0 9 0 1 )}$ & $\mathbf{0 . 0 0 1 2}(\mathbf{0 . 0 0 1 9})$ \\
\hline
\end{tabular}

Table 3. The percentage in different overlapping score intervals of different methods on the MESSIDOR dataset

\begin{tabular}{|c|c|c|c|c|}
\hline Methods & $\mathrm{JC} \geq 0.9$ & $\mathrm{JC} \geq 0.85$ & $\mathrm{JC} \geq 0.75$ & $\mathrm{~J} \geq 0.7$ \\
\hline Yu et al. [17] & 25 & 45 & 77 & 77 \\
Roychowdhury et al. [10] & 20 & 47.56 & 82.85 & $\mathbf{9 6 . 7 2}$ \\
Ours & $\mathbf{3 9 . 5 0}$ & $\mathbf{6 4 . 5 0}$ & $\mathbf{8 5 . 5 8}$ & 90.92 \\
\hline
\end{tabular}

They only take the grey channel into consideration but ignore the discriminativeness of the colour information. In the proposed method, the Lab colour feature and two background priors are considered, which may be the reason that the proposed method outperforms Morales et al. [21].

Table 3 shows the percentage in different overlapping score intervals of the proposed method and two previous methods on MESSIDOR dataset. The proposed method outperforms the other two methods when $J C \geq 0.75$. But the percentages of images the proposed method obtains in the interval $J C \geq 0.70$ is $90.92 \%$ which is inferior to Morales et al. [22] by $5.8 \%$. The reason may be that our method fails when there is large appearance difference between the superpixels in the OD.

\section{Conclusion}

This paper proposes a saliency-based OD segmentation method, in which the OD is regarded as the salient object. It includes two stages: the OD location stage and saliency-based OD segmentation stage. In the OD location stage, the candidate location is provided via filtering the grey channel of the retinal image with Laplacian of Gaussian filter and further validated by the vessel intensity. Then two priors are used to compute the saliency for OD segmentation. By directly thresholding the saliency map and fitting the segmented map with an ellipse, we obtain the final OD segment. The proposed method is completely automatic. It is simple but effective for optic disc segmentation. The experimental results on two public datasets show that our method achieves the stateof-the-art performances.

\section{References}

[1] D. Pascolini and S.P. Mariotti, "Global estimates of visual impairment", British Journal of Ophthalmology,doi: 10.1136/bjophthalmol-2011-300539, 2011.

[2] J. Cheng, F. Yin, D.W.K. Wong, et al., "Sparse dissimilarityconstrained coding for glaucoma screening “, IEEE Transactions on Biomedical Engineering, Vol.62, No.5, pp.1395-1403, 2015.

[3] A. Singh, M.K. Dutta, M. ParthaSarathi, et al., "Image processing based automatic diagnosis of glaucoma using wavelet features of segmented optic disc from fundus image", Computer methods and programs in biomedicine, Vol.124, pp.108120, 2016.

[4] K.S. Chin, E. Trucco, L. Tan, et al., "Automatic fovea location in retinal images using anatomical priors and vessel density", Pattern Recognition Letters, Vol.34, No.10, pp.1152-1158, 2013.

[5] Q. Liu, B. Zou, J. Chen, et al., "A location-to-segmentation strategy for automatic exudate segmentation in colour retinal fundus images", Computerized Medical Imaging and Graphics,Vol.55, pp.78-86, 2017.

[6] M. Lalonde, M. Beaulieu, and L. Gagnon, "Fast and robust optic disc detection using pyramidal decomposition and hausdorffbased template matching ", IEEE transactions on medical imaging, Vol.20, No.11, pp.1193-1200, 2001.

[7] J. Lowell, A. Hunter, D. Steel, et al., "Optic nerve head segmentation", IEEE Transactions on medical Imaging, Vol.23, No.2, pp.256-264, 2004

[8] Q. Liu, B. Zou, and J. Chen, et al., "Retinal vessel segmentation from simple to difficult", MICCAI workshop on ophthalmic medical image analysis, 2016.

[9] H. Eviatar, and R.L. Somorjai, "A fast, simple active contour algorithm for biomedical images ", Pattern Recognition Letters, Vol.17, No.9, pp.969-974, 1996.

[10] S. Roychowdhury, D.D. Koozekanani, and S.N. Kuchinka, et al. 
"Optic disc boundary and vessel origin segmentation of fundus images", IEEE journal of biomedical and health informatics, Vol.20, No.6, pp.1562-1574, 2016.

[11] G.D. Joshi, J. Sivaswamy, and S. Krishnadas, "Optic disk and cup segmentation from monocular color retinal images for glaucoma assessment",IEEE transactions on medical imaging, Vol.30, No.6, pp.1192-1205, 2011.

[12] J. Xu, O. Chutatape, and E. Sung, et al., "Optic disk feature extraction via modified deformable model technique for glaucoma analysis", Pattern recognition, Vol.40, No.7, pp.20632076, 2007.

[13] A. Salazar-Gonzalez, D. Kaba, Y. Li, et al., "Segmentation of the blood vessels and optic disk in retinal images", IEEE journal of biomedical and health informatics, Vol.18, No.6, pp.18741886, 2014.

[14] Y. Wei, F. Wen, W. Zhu, et al., "Geodesic saliency using background priors", European conference on computer vision, Springer, pp.29-42, 2012.

[15] B. Zou, Q. Liu, Z. Chen, et al., "Saliency detection using boundary information", Multimedia Systems, Vol.22, No.2, pp.245$253,2016$.

[16] J. Zhang, D. Zhao, and W. Gao, "Group-based sparse representation for image restoration", IEEE Transactions on Image Processing,Vol.23, No.8, pp.3336-3351, 2014.

[17] H. Yu, E.S. Barriga, C. Agurto, et al., "Fast localization and segmentation of optic disk in retinal images using directional matched filtering and level sets", IEEE Transactions on Information Technology in Biomedicine, Vol.16, No.4, pp.644-657, 2012 .

[18] R. Achanta, A. Shaji, K. Smith, et al., "Slic superpixels compared to state-of-the-art superpixel methods", IEEE transactions on pattern analysis and machine intelligence, Vol.34, No.11, pp.2274-2282, 2012.

[19] E.J. Carmona, M. Rincn, J. Garca-Feijo, et al., "Identification of the optic nerve head with genetic algorithms", Artificial Intelligence in Medicine, Vol.43, No.3, pp.243-259, 2008.

[20] E. Decencire, X. Zhang, G. Cazuguel, et al., "Feedback on a publicly distributed database:the messidor database", Image Analysis \& Stereology, Vol.33, No.3, pp.231-234, 2014.

[21] S. Morales, V. Naranjo, J. Angulo, et al., "Automatic detection of optic disc based on pca and mathematical morphology", IEEE transactions on medical imaging, Vol.32, No.4, pp.786796, 2013.

[22] T. Walter, J.C. Klein, P. Massin, et al., "A contribution of image processing to the diagnosis of diabetic retinopathy-detection of exudates in colour fundus images of the human retina ", IEEE transactions on medical imaging, Vol.21, No.10, pp.1236-1243, 2002 .

ZOU Beiji received the

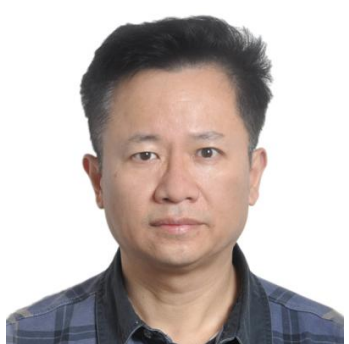
B.S., M.S., and Ph.D degrees from Zhejiang University in 1982 , Qinghua University in 1984 and Hunan University in 2001 respectively. He is currently a Professor and served as the dean at the School of Information Science and Engineering at Central South University. His research interests include computer graphics and image processing. (Email: bjzou@csu.edu.cn)

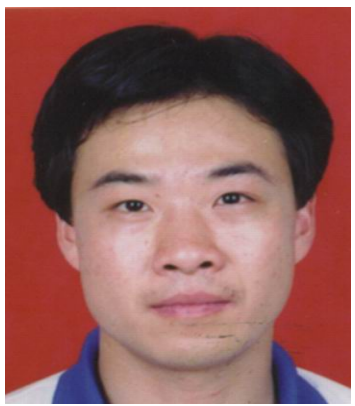

CHEN Zailiang (corresponding author) Chen Zailiang, received the Ph.D. degree in Computer Science from Central South University in 2012. He is currently an associate professor at the School of Information Science and Engineering, Central South University. His research interests include medical image analysis and saliency detection.(Email: zailiangchencs@gmail.com ) 